\title{
molecules
}

ISSN 1420-3049

http://www.mdpi.org

Full Paper

\section{A Convenient Approach to Heterocyclic Building Blocks: Synthesis of Novel Ring Systems Containing a [5,6]Pyrano[2,3-c]pyrazol-4(1H)-one Moiety}

\section{Gernot A. Eller * and Wolfgang Holzer ${ }^{\dagger}$}

Department of Drug and Natural Product Synthesis, Faculty of Life Sciences, University of Vienna, Althanstrasse 14, A-1090 Vienna, Austria; ${ }^{\dagger}$ E-mail: wolfgang.holzer@univie.ac.at

* Author to whom correspondence should be addressed. E-mail: gernot.eller@univie.ac.at

Received: 15 January 2007; in revised form: 24 January 2007 / Accepted: 24 January 2007 /

Published: 26 January 2007

\begin{abstract}
Starting from commercially available educts, a straightforward synthetic route to new heterocyclic building blocks is exemplified with the one- or two-step synthesis of tri-, tetra-, or pentacyclic ring systems. Representatives of the following novel ring systems are prepared from 3-methyl-1-phenyl-2-pyrazolin-5-one and the corresponding o-haloarenecarbonyl chloride using calcium hydroxide in refluxing 1,4-dioxane: pyrimidino[4',5':5,6]pyrano[2,3-c]pyrazol-4(1H)-one, thieno[3',2':5,6]pyrano[2,3c]pyrazol4-(1H)-one, thieno[3',4':5,6]pyrano[2,3-c]pyrazol-4(1H)-one, thieno[3",2":4',5']thieno[2',3':5,6]pyrano[2,3-c]pyrazol-4(1H)-one, [1,3]dioxolo[5',6'][1]benzothieno[2',3':5,6]pyrano[2,3-c]pyrazol-4(1H)-one, pyridazino[4',3':5,6]pyrano[2,3-c]pyrazol-4(1H)-one, and pyrazolo[4",3":5',6']pyrido[3',4':5,6]pyrano[2,3-c]pyrazol-6(9H)-one. While the latter two ring systems are directly obtained due to a spontaneous intramolecular substitution reaction, in the other reactions uncyclised 4-aroylpyrazol-5-ols are produced, which are cyclised into the target heterocycles in a subsequent synthetic step (i.e. treatment with $\mathrm{NaH}$ in DMF). Detailed NMR spectroscopic investigations $\left({ }^{1} \mathrm{H}-,{ }^{13} \mathrm{C}-,{ }^{15} \mathrm{~N}-\right)$ with the obtained compounds were undertaken to unambiguously prove the new structures.
\end{abstract}

Keywords: Fused-ring systems, NMR spectroscopy, heterocyclic building blocks, acylations, pyrazolones. 


\section{Introduction}

The search for biologically active compounds is the driving force in pharmaceutical synthesis. Since the majority of new molecules entering clinical studies contains at least one heterocyclic moiety - predominantly N-heterocyclic ones [1] - the modification of these ring systems plays an important role during drug development [2,3]. Thus, there is always a specific need for novel heterocyclic ring systems, both for finding new hit structures and in optimisation of lead compounds. Although theoretically unlimited, in practical terms and due to technical and economical reasons, only a very limited number of heterocycles is available for medicinal chemistry today.

\section{Figure 1.}<smiles>O=c1c2ccccc2oc2ccccc12</smiles><smiles>CC(C)c1ccc2oc3nc(N)c(C(=O)O)cc3c(=O)c2c1</smiles>

xanthone

(dibenzo- $\gamma$-pyrone)

amlexanox

(Aphthasol ${ }^{\mathrm{TM}}$ )<smiles>Cc1nn(-c2cccc(N)c2)c2oc3ccccc3c(=O)c12</smiles>

A

Our own interest in new and easily accessible heterocyclic building blocks stems from our ongoing research on xanthone (= dibenzo- $\gamma$-pyrone) derivatives, in which one of the benzene rings is replaced with a pyrazole nucleus and the other with a different heterocyclic moiety [4-6]. These interesting substructures occur in several bioactive compounds, such as in the anti-ulcer drug, amlexanox (Aphthasol ${ }^{\mathrm{TM}}$ ) [7,8] or in the $\mathrm{A}_{2}$-subtype selective adenosine receptor antagonist $\mathbf{A}$ [9] (Figure 1). Consequently we investigated several synthetic strategies to facilitate alteration of this biologically interesting scaffold. While our primary research was based on synthetic approaches for the convenient variation of the substituents at the pyrazole core (in particular those of positions $\mathrm{C}-3, \mathrm{~N}-1$ and N-2) $[10,11]$, we turned our interest to the modification of the molecule's skeleton as well as the possibility of introducing substituents at other positions. The combination of these approaches would obviously allow access to specifically customised molecules. Nevertheless, only a few skeletons - mainly tricyclic ones like the possible four pyridines [4] - have been reported as successfully attempted up to the present.

\section{Results and Discussion}

The purpose of this study is to report the convenient one- or two-step synthesis of novel heterocyclic ring systems (Table 1 ) containing a [5,6]pyrano[2,3-c]pyrazol-4(1H)-one moiety starting from commercially available educts. Thus, 2-pyrazolin-5-one 1 (a tautomer of 5-hydroxypyrazole [12]) was reacted with the corresponding o-haloarenecarbonyl chlorides $\mathbf{2 b}-\mathbf{8 b}$ (Figure 2) in the presence of excess calcium hydroxide in boiling 1,4-dioxane (the 'Jensen' method [13]) to affect the selective acylation at the pyrazole C-4. However, the target compounds $\mathbf{9}$ and $\mathbf{1 0}$ were directly 
obtained in the reaction of the acid chlorides $\mathbf{2} \mathbf{b}$ and $\mathbf{3 b}$ with pyrazolone $\mathbf{1}$, without isolation of the expected 4-aroylpyrazol-5-ols (Scheme 1).

Figure 2. Structure of $o$-haloarenecarbonyl chlorides $\mathbf{2 b - 8 b}$.<smiles>[Y]C(=O)c1cc(Cl)nnc1Cl</smiles>

2<smiles>[Y]C(=O)c1nc(SC)ncc1Cl</smiles>

4<smiles>[Y]C(=O)c1cscc1Br</smiles>

6 7b
$8 b$

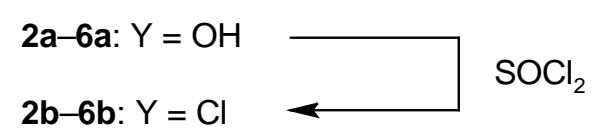

Obviously, spontaneous intramolecular cyclisation did occur (only) in these two cases, leading to formation of two new bonds ( $\mathrm{C}-\mathrm{C}$ and $\mathrm{O}-\mathrm{C}$, respectively) in a single reaction step in these molecules. This fact could be correlated with the activity towards nucleophilc aromatic substitution of the leaving halogen in the presumptive intermediates 21 and 22 (Figure 3). In electron-rich aromatic compounds (which are known to be less reactive towards nucleophilic aromatic substitutions) such as thiophene derivatives 17-20 or even in the pyrimidine example $\mathbf{1 6}$ (where the halogen atom is situated meta to the activating $\mathrm{N}$ atom and hence the least active position in such pyridines), no cyclisation was observed. However, ring closure occurred spontaneously in the reactions of the acid chlorides $\mathbf{2} \mathbf{b}$ and $\mathbf{3 b}$, in which the halogen was situated ortho or para to the activating $\mathrm{N}$ atom. This behaviour is in full agreement with the observations made in cyclisation reactions leading to different pyridino[5,6]pyrano[2,3-c]pyrazol4(1H)-ones [4].

Scheme 1. Synthesis of the title compounds.<smiles>Cc1nn(-c2ccccc2)c2c1C(=O)C1CCCCC1O2</smiles>

9, 10<smiles>CC1=NN(c2ccccc2)C(=O)C1</smiles>

1

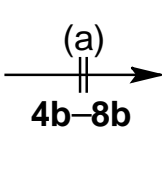

(a)
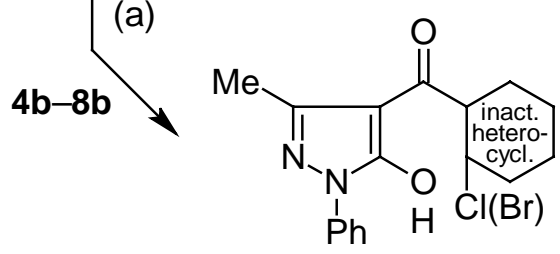<smiles>Cc1nn(-c2ccccc2)c2c1C(=O)C1CCCCC1O2</smiles>

11-15

\section{(b) $\uparrow$}

$16-20$

(a) $o$-haloarenecarbonyl chloride (2b-8b), $\mathrm{Ca}(\mathrm{OH})_{2}$, 1,4-dioxane. (b) $\mathrm{NaH}, \mathrm{DMF}$. 
When the uncyclised intermediates were isolated, the transformation into the target ring systems was achieved by treatment of 4-aroylpyrazol-5-ols 16-20 with sodium hydride in refluxing dimethylformamide.

Figure 3. Intermediate 4-Aroylpyrazol-5-ols 16-22.<smiles>Cc1nn(-c2ccccc2)c(O)c1C(=O)c1cc(Cl)nnc1Cl</smiles>

21*<smiles>Cc1nn(-c2ccccc2)c(O)c1C(=O)c1cnc2c(c(C)nn2C)c1Cl</smiles>

22*<smiles></smiles>

16

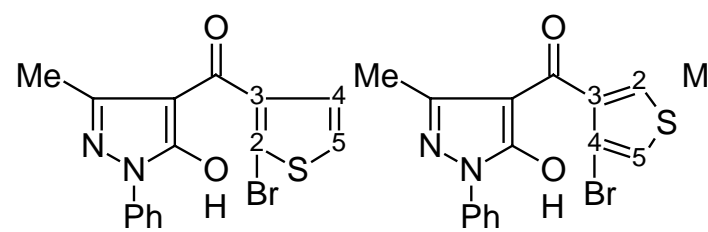

17<smiles></smiles>

19

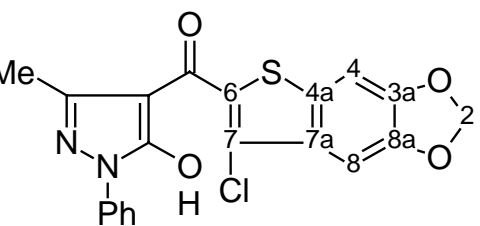

20

* not isolated.

\section{NMR spectroscopy}

Recently, assignment of NMR signals (mainly ${ }^{13} \mathrm{C}-\mathrm{NMR}$ lines) using prediction programs [14] such as CSEARCH [15] or ACD/C+H NMR Predictor [16] has become increasingly popular. However, the quality of such empirical predictions strongly depends on the availability in the corresponding databases of reliable material data from related structures. Thus, in cases where insufficient (or low quality) data is available for the description of a specific chemical environment, the prediction results are poor. The latter seems to be the case for many condensed heterocyclic systems containing few protons. This lack of high quality data provided the motivation for us to perform detailed NMR spectroscopic investigations of all novel compounds prepared and also their precursors. Full and unambiguous assignment for all proton $\left({ }^{1} \mathrm{H}-\right)$, carbon $\left({ }^{13} \mathrm{C}-\right)$, and nitrogen $\left({ }^{15} \mathrm{~N}-\right)$ resonances could be achieved by the combined application of different NMR spectroscopic techniques [17] such as NOESY [18], NOE-difference experiments $\left({ }^{1} \mathrm{H}\left\{{ }^{1} \mathrm{H}\right\}\right.$ [18] as well as ${ }^{13} \mathrm{C}\left\{{ }^{1} \mathrm{H}\right\}$ [18-20]), fully ${ }^{1} \mathrm{H}$-coupled ${ }^{13} \mathrm{C}$-NMR spectra, APT, HMQC, gs-HMSC, and gs-HMBC spectra. The assignments were also be facilitated by experiments with selective excitation such as 1D-TOCSY [21], 1DHETCOR [22], and selective long-range INEPT experiments [23]. ${ }^{13} \mathrm{C},{ }^{1} \mathrm{H}$ spin coupling constants were also determined and unequivocally assigned by two-dimensional $(\delta, J)$ long-range INEPT spectra with selective excitation [24]. The ${ }^{15} \mathrm{~N}-\mathrm{NMR}$ spectra were mainly recorded using the gradient selected, sensitivity enhanced HMBC sequence [25].

The assignment of the numerous lines due to quaternary carbon atoms has not been a trivial task. For instance, in target compounds 9-15, the chemical shifts of the two carbon atoms directly attached to the pyran oxygen atom often closely resemble each other. Despite this fact, an unequivocal distinction was possible on basis of the ${ }^{1} \mathrm{H}$-coupled ${ }^{13} \mathrm{C}$-NMR spectra. The signal of the carbon atom located between pyrazole $\mathrm{N}-1$ and the pyran oxygen always appears as a singlet, whereas the signal of 
the opposite pyran $\mathrm{O}-\mathrm{C}$ atom is split by couplings to protons belonging to the system condensed on the 'right' side of the pyran ring. In compound 14 this diagnostic coupling is small $\left({ }^{3} J_{\mathrm{C} 8 \mathrm{~b}, \mathrm{H} 8}=1.0 \mathrm{~Hz}\right)$ and emerges only after pronounced resolution enhancement. Discrimination of C-5a vs. C-8a in 14, C-5a vs. C-10a in 15, and C-5 vs. C-7 in 13 is based on the upfield shift of the latter signal in each pair due to the influence of the pyran oxygen atom. In the fused pyrimidine derivative $\mathbf{1 1}$ the discrimination of the lines due to C-4a and C-8a (144.4 ppm, $146.0 \mathrm{ppm}$ ) is not possible considering the ${ }^{1} \mathrm{H}$-coupled ${ }^{13} \mathrm{C}$ NMR spectrum, since both signals show a $3 \mathrm{~Hz}$ splitting due to coupling with $\mathrm{H}-8$. Here, unambiguous assignment of C-8a was achieved via a heteronuclear ${ }^{13} \mathrm{C}\left\{{ }^{1} \mathrm{H}\right\}$ NOE-difference experiment. Irradiation of the H-8 transition only enhanced the signal of the spatially close C-8a whereas the line of the more distant C-4a remained unaffected (Figure 3).

Figure 3. Unambiguous ${ }^{13} \mathrm{C}-\mathrm{NMR}$ signal assignment of C-4a and C-8 in compound $\mathbf{1 1}$ in $\mathrm{CDCl}_{3}:{ }^{13} \mathrm{C}\left\{{ }^{1} \mathrm{H}\right\}$-NOE on C-8 and C-8a upon selective irradiation of H-8.
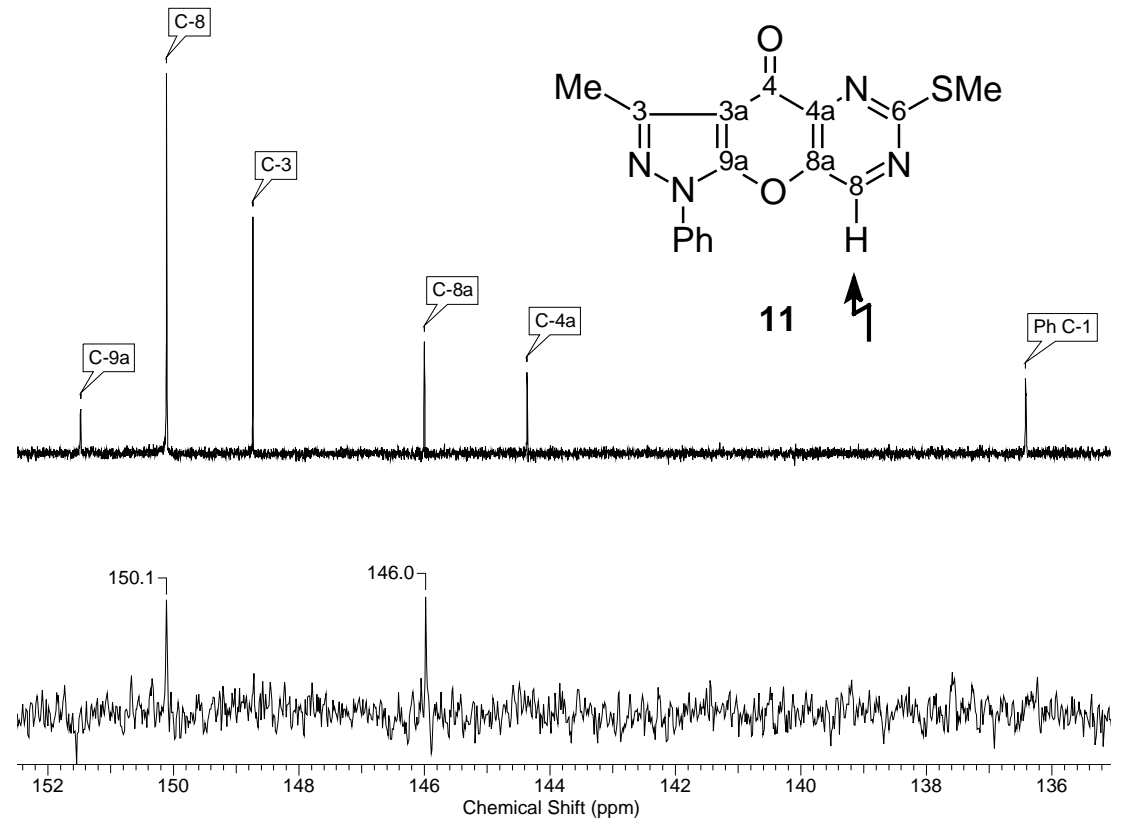

A similar approach was used to support the discrimination of the signals due to C-3a and C-6a in 4acylpyrazolone 19 (enhancement of the C-3a signal after irradiation of $\mathrm{H}-4$ ). In the latter compound, the signal of $\mathrm{H}-4$ (attached to the carbon atom in $\beta$-position to the thiophene S-atom 6) can be simply distinguished from that of $\mathrm{H}-5$ (attached to $\mathrm{C}-5$, in $\alpha$-position to S-6) considering the larger ${ }^{1} J$ coupling constants in the thiophene $\alpha-\mathrm{C}, \mathrm{H}$ fragment (in 19: ${ }^{1} J_{\mathrm{C} 5, \mathrm{H} 5}=189.3 \mathrm{~Hz}>{ }^{1} J_{\mathrm{C} 4, \mathrm{H} 4}=173.3 \mathrm{~Hz}$ ). This criterion was also employed to achieve correct assignments in compounds 7b, 12, 14, and 17.

In most cases, assignment of ${ }^{15} \mathrm{~N}$-NMR signals was easily made on basis of chemical shift considerations together with correlations emerging from the gs-HMBC spectra. As an illustrative example, the ${ }^{15} \mathrm{~N},{ }^{1} \mathrm{H}$ HMBC spectrum of compound $\mathbf{1 0}$ is displayed in Figure 4. 
Figure 4. ${ }^{15} \mathrm{~N},{ }^{1} \mathrm{H}-\mathrm{HMBC}$ spectrum of compound $\mathbf{1 0}$ in $\mathrm{CDCl}_{3}$.

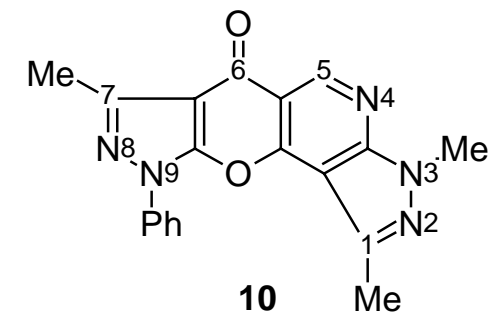

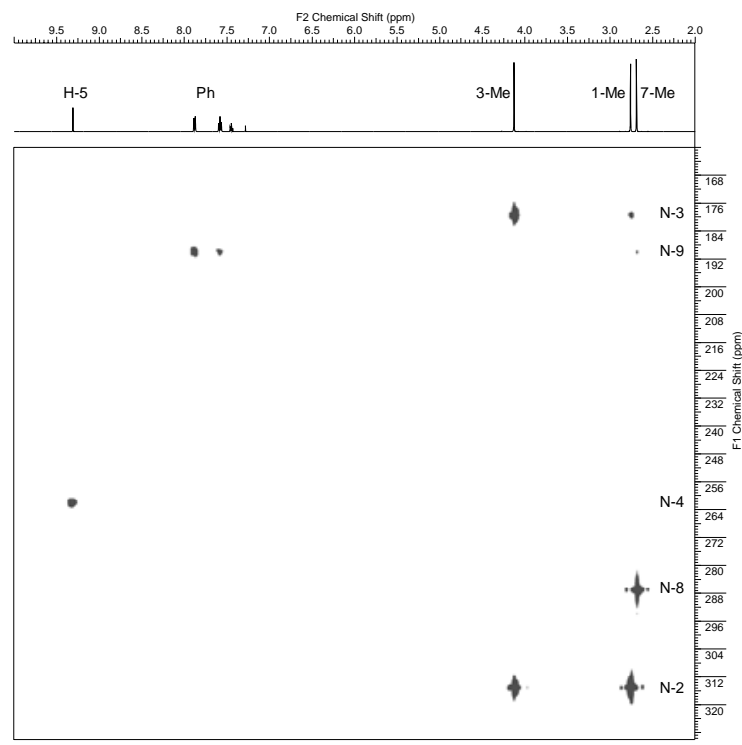

Table 1. ${ }^{1} \mathrm{H}$-, ${ }^{13} \mathrm{C}$-, and ${ }^{15} \mathrm{~N}$-NMR chemical shifts as well as selected ${ }^{1} \mathrm{H},{ }^{1} \mathrm{H}$ and ${ }^{13} \mathrm{C},{ }^{1} \mathrm{H}$ Spin-Coupling Constants of the Novel Ring Systems 9-15.

\begin{tabular}{|c|c|c|}
\hline Entry & Structure & NMR data \\
\hline 9 & & $\begin{array}{l}{ }^{1} \mathrm{H}-\mathrm{NMR}\left(500 \mathrm{MHz}, \mathrm{CDCl}_{3}\right) \delta: 2.66(3 \mathrm{H}, \mathrm{s}, \mathrm{Me}), 7.43(1 \mathrm{H}, \mathrm{m}, \mathrm{Ph} \\
\mathrm{H}-4), 7.56(2 \mathrm{H}, \mathrm{m}, \mathrm{Ph} \mathrm{H}-3,5), 7.92(2 \mathrm{H}, \mathrm{m}, \mathrm{Ph} \mathrm{H}-2,6), 8.36(1 \mathrm{H}, \mathrm{s}, \\
\mathrm{H}-5) . \\
{ }^{13} \mathrm{C}-\mathrm{NMR}\left(125 \mathrm{MHz}, \mathrm{CDCl}_{3}\right) \delta: 14.0\left(\mathrm{Me},{ }^{1} \mathrm{~J}=129.7 \mathrm{~Hz}\right), 105.1(\mathrm{C}- \\
\left.3 \mathrm{a},{ }^{3} J_{\mathrm{C} 3, \mathrm{Me}}=2.8 \mathrm{~Hz}\right), 121.6(\mathrm{Ph} \mathrm{C}-2,6), 122.7\left(\mathrm{C}-4 \mathrm{a},{ }^{2} J_{\mathrm{C} 4 \mathrm{a}, \mathrm{H} 5}=1.3 \mathrm{~Hz}\right), \\
126.9\left(\mathrm{C}-5,{ }^{1} \mathrm{~J}=179.0 \mathrm{~Hz}\right), 128.3(\mathrm{Ph} \mathrm{C}-4), 129.7(\mathrm{Ph} \mathrm{C}-3,5), 136.1 \\
(\mathrm{Ph} \mathrm{C}-1), 148.7\left(\mathrm{C}-3,{ }^{2} J_{\mathrm{C} 3, \mathrm{Me}}=7.2 \mathrm{~Hz}\right), 152.2(\mathrm{C}-9 \mathrm{a}), 155.5(\mathrm{C}-6, \\
\left.{ }^{2} J_{\mathrm{C} 6, \mathrm{H} 5}=0.9 \mathrm{~Hz}\right), 160.3 \quad\left(\mathrm{C}-8 \mathrm{a},{ }^{3} J_{\mathrm{C} 8 \mathrm{a}, \mathrm{H} 5}=7.1 \mathrm{~Hz}\right), 169.7(\mathrm{C}-4, \\
\left.{ }^{3} J_{\mathrm{C} 4 \mathrm{H} 5}=4.3 \mathrm{~Hz}\right) . \\
{ }^{15} \mathrm{~N}-\mathrm{NMR}(50 \mathrm{MHz}, \mathrm{CDCl} 3) \delta:-189.5(\mathrm{~N}-1),-89.6(\mathrm{~N}-2),-8.6(\mathrm{~N}- \\
8), 19.9(\mathrm{~N}-7) .\end{array}$ \\
\hline
\end{tabular}


Table 1. Cont.

\begin{tabular}{|c|c|c|}
\hline Entry & Structure & NMR data \\
\hline 10 & $\mathrm{Me}$ & 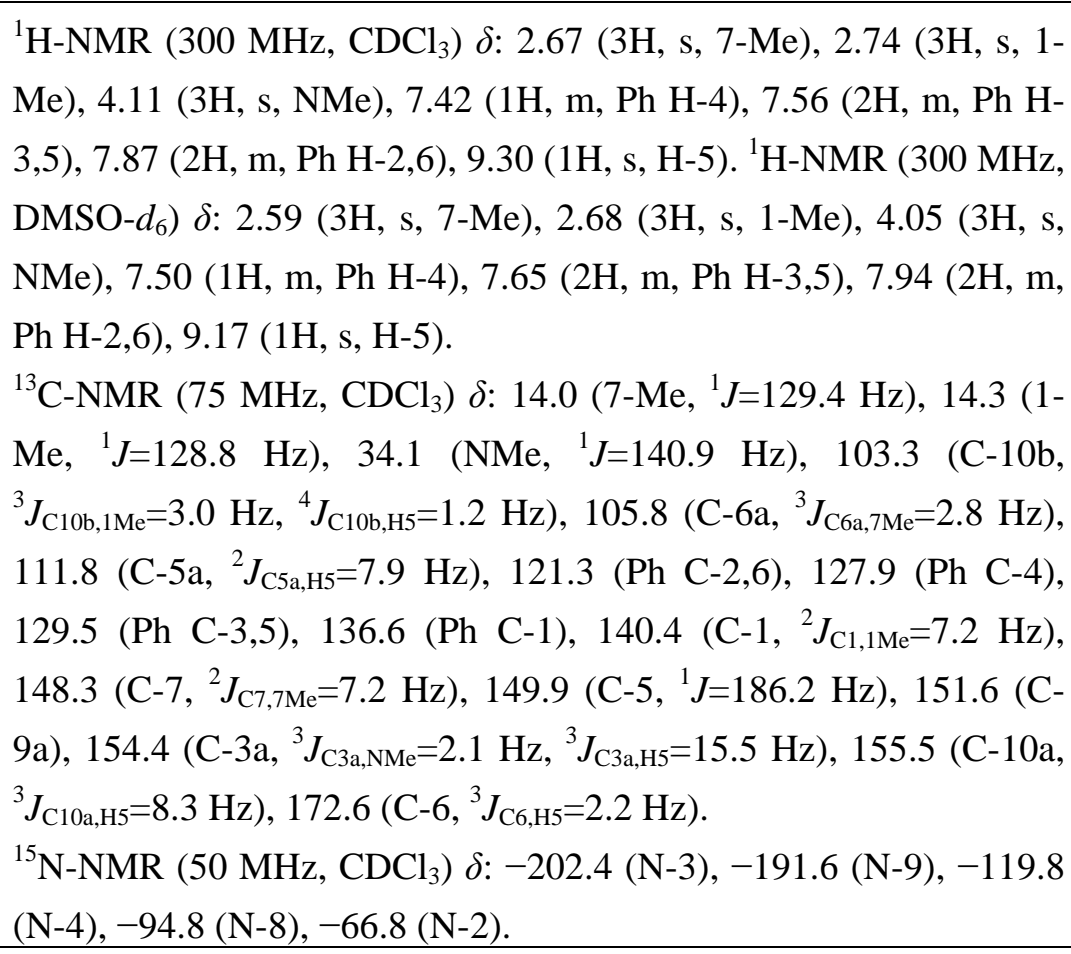 \\
\hline 11 & $\pi \pi$ & $\begin{array}{l}{ }^{1} \mathrm{H}-\mathrm{NMR}\left(300 \mathrm{MHz}, \mathrm{CDCl}_{3}\right) \delta: 2.68(3 \mathrm{H}, \mathrm{s}, 3-\mathrm{Me}), 2.68(3 \mathrm{H}, \mathrm{s}, \\
\mathrm{SMe}), 7.42(1 \mathrm{H}, \mathrm{m}, \mathrm{Ph} \mathrm{H}-4), 7.55(2 \mathrm{H}, \mathrm{m}, \mathrm{Ph} \mathrm{H}-3,5), 7.82(2 \mathrm{H}, \mathrm{m}, \\
\mathrm{Ph} \mathrm{H}-2,6), 8.96(1 \mathrm{H}, \mathrm{s}, \mathrm{H}-8) . \\
{ }^{13} \mathrm{C}-\mathrm{NMR}\left(75 \mathrm{MHz}, \mathrm{CDCl}_{3}\right) \delta: 14.1\left(3-\mathrm{Me},{ }^{1} \mathrm{~J}=129.6 \mathrm{~Hz}\right), 14.8 \\
\left(\mathrm{SMe},{ }^{1} J=141.8 \mathrm{~Hz}\right), 107.8\left(\mathrm{C}-3 \mathrm{a},{ }^{3} J_{\mathrm{C} 3 \mathrm{a}, 3 \mathrm{Me}}=2.8 \mathrm{~Hz}\right), 121.5(\mathrm{Ph} \mathrm{C}- \\
2,6), 128.0(\mathrm{Ph} \mathrm{C}-4), 129.6(\mathrm{Ph} \mathrm{C}-3,5), 136.4(\mathrm{Ph} \mathrm{C}-1), 144.4(\mathrm{C}-4 \mathrm{a}, \\
\left.{ }^{3} J_{\mathrm{C} 4 \mathrm{a}, \mathrm{H} 8}=3.0 \mathrm{~Hz}\right), 146.0\left(\mathrm{C}-8 \mathrm{a},{ }^{2} J_{\mathrm{C} 8 \mathrm{a}, \mathrm{H} 8}=3.0 \mathrm{~Hz}\right), 148.8(\mathrm{C}-3 \text {, } \\
\left.{ }^{2} J_{\mathrm{C} 3,3 \mathrm{Me}}=7.2 \mathrm{~Hz}\right), 150.1\left(\mathrm{C}-8,{ }^{1} \mathrm{~J}=187.4 \mathrm{~Hz}\right), 151.5(\mathrm{C}-9 \mathrm{a}), 170.2(\mathrm{C}- \\
\left.{ }^{6},{ }^{3} J_{\mathrm{C} 6 \mathrm{SMe}}=4.7 \mathrm{~Hz},{ }^{3} J_{\mathrm{C} 6, \mathrm{H} 8}=12.8 \mathrm{~Hz}\right), 170.7\left(\mathrm{C}-4,{ }^{4} J_{\mathrm{C} 4, \mathrm{H} 8}=2.0 \mathrm{~Hz}\right) . \\
{ }^{15} \mathrm{~N}-\mathrm{NMR}(50 \mathrm{MHz}, \mathrm{CDCl}) \delta:-191.9(\mathrm{~N}-1),-108.9(\mathrm{~N}-5),-91.1 \\
(\mathrm{~N}-2),-80.2(\mathrm{~N}-7) .\end{array}$ \\
\hline 12 & & 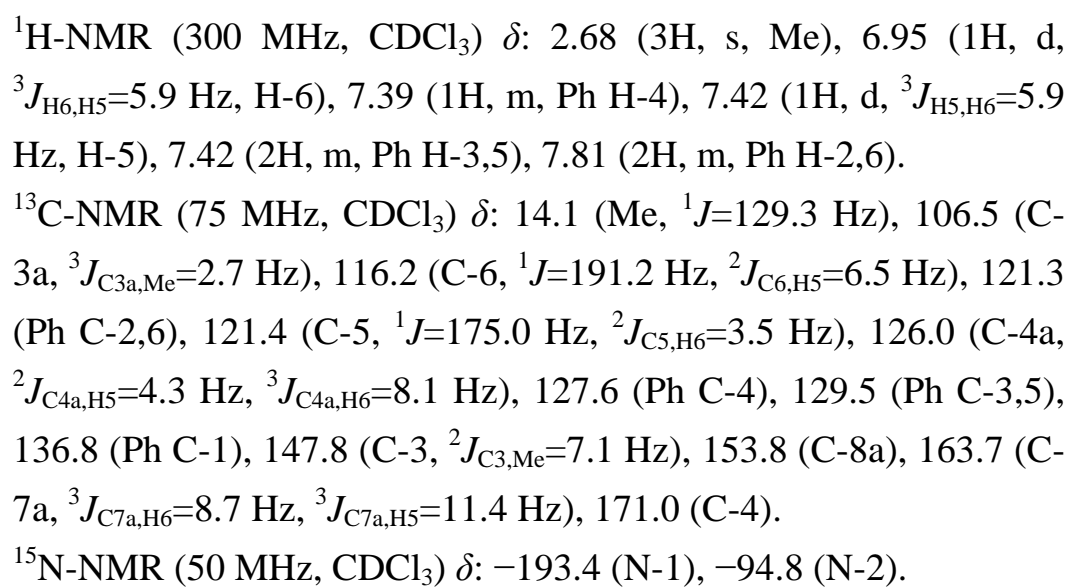 \\
\hline
\end{tabular}


Table 1. Cont.

\begin{tabular}{|c|c|c|}
\hline Entry & Structure & NMR data \\
\hline 13 & & $\begin{array}{l}{ }^{1} \mathrm{H}-\mathrm{NMR}\left(500 \mathrm{MHz}, \mathrm{CDCl}_{3}\right) \delta: 2.70(3 \mathrm{H}, \mathrm{s}, \mathrm{Me}), 7.15(1 \mathrm{H}, \mathrm{d}, \\
\left.{ }^{3} J_{\mathrm{H} 7, \mathrm{H} 5}=3.3 \mathrm{~Hz}, \mathrm{H}-7\right), 7.40(1 \mathrm{H}, \mathrm{m}, \mathrm{Ph} \mathrm{H}-4), 7.54(2 \mathrm{H}, \mathrm{m}, \mathrm{Ph} \mathrm{H}-3,5), \\
7.85(2 \mathrm{H}, \mathrm{m}, \mathrm{Ph} \mathrm{H}-2,6), 8.12\left(1 \mathrm{H}, \mathrm{d},{ }^{3} J_{\mathrm{H} 5, \mathrm{H} 7}=3.3 \mathrm{~Hz}, \mathrm{H}-5\right) . \\
{ }^{13} \mathrm{C}-\mathrm{NMR}\left(125 \mathrm{MHz}, \mathrm{CDCl}{ }_{3}\right) \delta: 14.2\left(\mathrm{Me},{ }^{1} \mathrm{~J}=129.3 \mathrm{~Hz}\right), 104.8(\mathrm{C}- \\
\left.3 \mathrm{a},{ }^{3} J_{\mathrm{C} 3 \mathrm{a}, \mathrm{Me}}=2.5 \mathrm{~Hz}\right), 105.7\left(\mathrm{C}-7,{ }^{1} \mathrm{~J}=190.0 \mathrm{~Hz},{ }^{3} J_{\mathrm{C}, \mathrm{H} 5}=4.3 \mathrm{~Hz}\right), 121.6 \\
(\mathrm{Ph} \mathrm{C}-2,6), 126.3\left(\mathrm{C}-5,{ }^{1} \mathrm{~J}=192.2 \mathrm{~Hz},{ }^{3} J_{\mathrm{C} 5, \mathrm{H} 7}=5.9 \mathrm{~Hz}\right), 127.5(\mathrm{Ph} \mathrm{C}- \\
\left.{ }^{4}\right), 129.4(\mathrm{Ph} \mathrm{C}-3,5), 129.5(\mathrm{C}-4 \mathrm{a}), 137.0(\mathrm{Ph} \mathrm{C}-1), 148.7(\mathrm{C}-3, \\
\left.{ }^{2} J_{\mathrm{C} 3, \mathrm{Me}}=7.1 \mathrm{~Hz}\right), 151.4\left(\mathrm{C}-7 \mathrm{a},{ }^{3} \mathrm{JC}_{7 \mathrm{a}, \mathrm{H} 5}=11.2 \mathrm{~Hz}\right), 154.7(\mathrm{C}-8 \mathrm{a}), 171.2 \\
\left(\mathrm{C}-4,{ }^{3} J_{\mathrm{C} 4, \mathrm{H} 5}=2.1 \mathrm{~Hz}\right) .\end{array}$ \\
\hline 14 & & 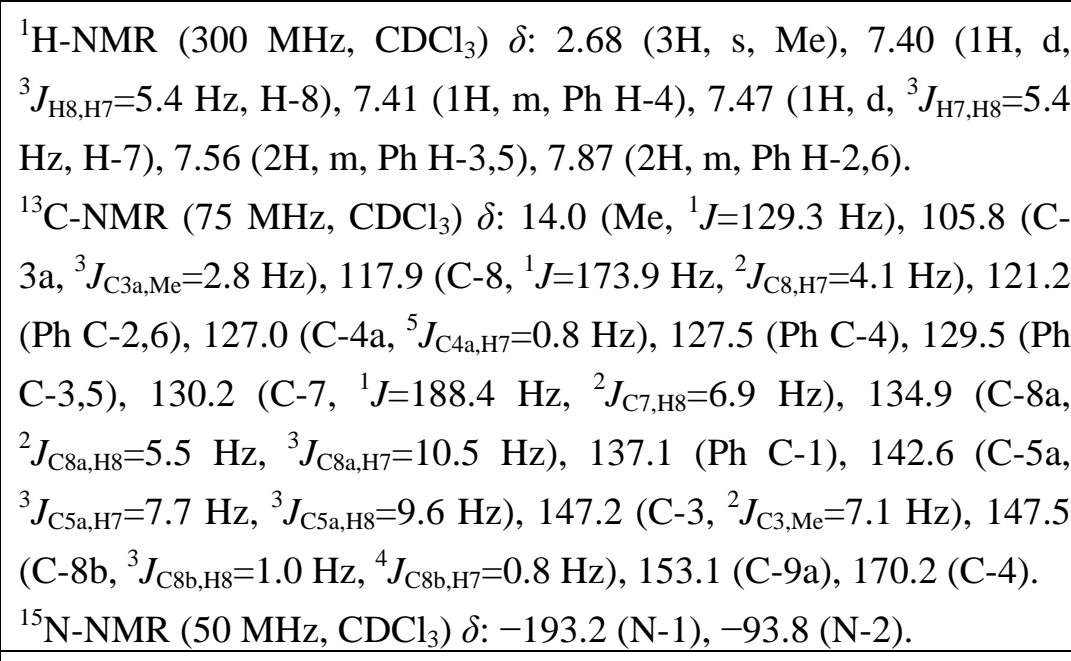 \\
\hline 15 & $\mathrm{Ph}$ & 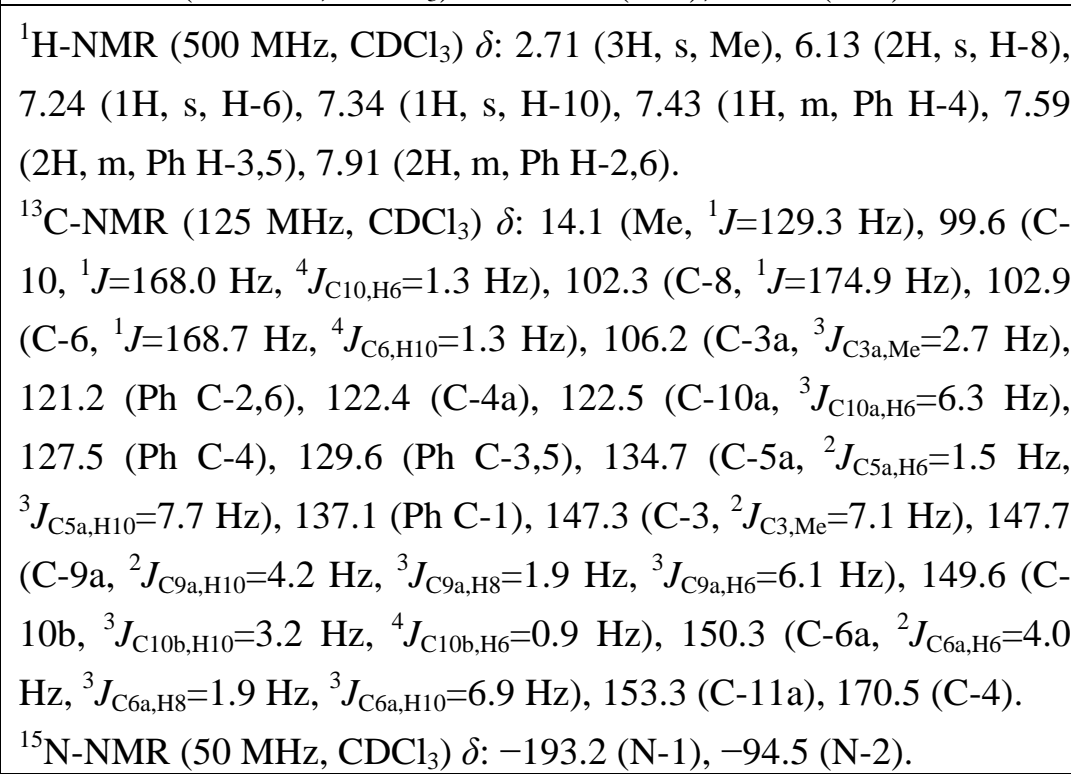 \\
\hline
\end{tabular}

\section{Experimental}

Materials and Methods

Melting points were determined on a Reichert-Kofler hot-stage microscope and are uncorrected. Mass spectra were obtained on a Shimadzu QP 1000 instrument (EI, $70 \mathrm{eV}$ ) and on a Finnigan MAT 
8230 instrument (EI, 70 eV, HRMS). IR spectra were recorded on a Perkin-Elmer FTIR Spectrum 1000 spectrometer. Elemental analyses were performed at the Microanalytical Laboratory, University of Vienna. ${ }^{1} \mathrm{H}$ - and ${ }^{13} \mathrm{C}$-NMR spectra were recorded on a Varian UnityPlus 300 spectrometer at $28{ }^{\circ} \mathrm{C}$ (299.95 MHz for ${ }^{1} \mathrm{H}, 75.43 \mathrm{MHz}$ for ${ }^{13} \mathrm{C}$ ) or on a Bruker Avance 500 spectrometer at $293 \mathrm{~K}$ (500.13 $\mathrm{MHz}$ for ${ }^{1} \mathrm{H}, 125.77 \mathrm{MHz}$ for ${ }^{13} \mathrm{C}$ ). The centre of the solvent signal was used as an internal standard which was related to TMS with $\delta=7.26 \mathrm{ppm}\left({ }^{1} \mathrm{H}\right.$ in $\left.\mathrm{CDCl}_{3}\right), \delta=2.49 \mathrm{ppm}\left({ }^{1} \mathrm{H}\right.$ in DMSO- $\left.d_{6}\right), \delta=77.0$ ppm $\left({ }^{13} \mathrm{C}\right.$ in $\left.\mathrm{CDCl}_{3}\right)$, and $\delta=39.5 \mathrm{ppm}\left({ }^{13} \mathrm{C}\right.$ in DMSO- $\left.d_{6}\right)$. The digital resolutions were $0.2 \mathrm{~Hz} /$ data point in the ${ }^{1} \mathrm{H}$ and $0.4 \mathrm{~Hz}$ /data point in the ${ }^{1} \mathrm{H}$-coupled ${ }^{13} \mathrm{C}$-NMR spectra (gated decoupling). ${ }^{15} \mathrm{~N}$ NMR spectra were obtained on a Bruker Avance 500 instrument with a 'directly' detecting broadband observe probe and were referenced against external nitromethane (coaxial capillary). Systematic names were generated with ACD/Name [26] according to the IUPAC recommendations and were also checked manually to ensure correct use of nomenclature within this publication [27]. All starting materials are commercially available and were purchased from Aldrich (1, 2a, 4a, 5a), Fluorochem (8b), Frontier Scientific (6a), or Maybridge (3a, 7b), respectively. Product yields were not optimised.

\section{o-Haloarenecarbonyl Chlorides 2b-6b: General Procedure.}

A suspension of the commercially available $o$-haloarenecarboxylic acid $\mathbf{2 a}$, 3a, $4 \mathbf{a}, \mathbf{5 a}$, or $\mathbf{6 a}$ (3 mmol) in toluene (10 mL), DMF (1 drop), and excess $\mathrm{SOCl}_{2}(2 \mathrm{~mL}$ ) were refluxed for $3 \mathrm{~h}$. The solvent and excess $\mathrm{SOCl}_{2}$ were removed under reduced pressure. Additional toluene $(5 \mathrm{~mL})$ was added and the solvent was removed under reduced pressure. The remaining acid chlorides of type $\mathbf{b}$ were used immediately, without further purification, in the next step. Commercially available acid chlorides $\mathbf{7 b}$ and $\mathbf{8 b}$ were used as purchased. Hitherto unpublished spectroscopic data of commercially available starting materials are presented below.

3,6-Dichloropyridazine-4-carboxylic acid (2a). ${ }^{1} \mathrm{H}-\mathrm{NMR}\left(300 \mathrm{MHz}, \mathrm{DMSO}-d_{6}\right) \delta: 8.28(1 \mathrm{H}, \mathrm{s}, \mathrm{H}-5)$, $9.72(1 \mathrm{H}, \mathrm{s}, \mathrm{OH}) ;{ }^{13} \mathrm{C}-\mathrm{NMR}\left(75 \mathrm{MHz}, \mathrm{DMSO}-d_{6}\right) \delta: 130.1\left(\mathrm{C}-5,{ }^{1} \mathrm{~J}=181.3 \mathrm{~Hz}\right), 134.0\left(\mathrm{C}-4,{ }^{2} J_{\mathrm{C} 4, \mathrm{H} 5}=1.0\right.$ $\mathrm{Hz}), 152.0\left(\mathrm{C}-3,{ }^{3} J_{\mathrm{C} 3, \mathrm{H} 5}=7.5 \mathrm{~Hz}\right), 156.2\left(\mathrm{C}-6,{ }^{2} J_{\mathrm{C} 6, \mathrm{H} 5}=1.3 \mathrm{~Hz}\right), 163.0\left(\mathrm{CO},{ }^{3} J_{\mathrm{CO}, \mathrm{H} 5}=4.8 \mathrm{~Hz}\right) ; \mathrm{MS} \mathrm{m} / \mathrm{z}$

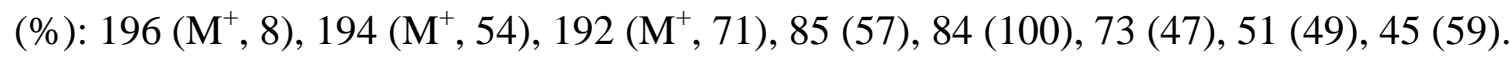

4-Chloro-1,3-dimethyl-1H-pyrazolo[3,4-b]pyridine-5-carboxylic acid (3a). ${ }^{1} \mathrm{H}-\mathrm{NMR}$ (300 MHz, DMSO- $\left.d_{6}\right) \delta$ : 2.36 (3H, s, 3-Me), 3.32* (1H, br s, OH), 3.96 (3H, s, NMe), $8.85(1 \mathrm{H}, \mathrm{s}, \mathrm{H}-6)$ * together with trace $\mathrm{H}_{2} \mathrm{O} ;{ }^{13} \mathrm{C}-\mathrm{NMR}$ (75 MHz, DMSO- $d_{6}$ ) $\delta$ : 14.5 (3-Me, ${ }^{1} \mathrm{~J}=128.9 \mathrm{~Hz}$ ), 33.6 (NMe, ${ }^{1} \mathrm{~J}=140.9$ $\mathrm{Hz}), 112.6$ (C-3a), 118.5 (C-5, $\left.{ }^{2} J_{\mathrm{C} 5, \mathrm{H} 6}=8.2 \mathrm{~Hz}\right), 138.7$ (C-4, $\left.{ }^{3} J_{\mathrm{C} 4, \mathrm{H} 6}=9.1 \mathrm{~Hz}\right), 141.3\left(\mathrm{C}-3,{ }^{2} J_{\mathrm{C} 3,3 \mathrm{Me}}=7.1\right.$ $\mathrm{Hz}$ ), 151.2 (C-6, ${ }^{1} \mathrm{~J}=186.2 \mathrm{~Hz}$ ), 151.5 (C-7a, ${ }^{3} J_{\mathrm{C} 7 \mathrm{a}, \mathrm{NMe}}=2.3 \mathrm{~Hz},{ }^{3} J_{\mathrm{C} 7 \mathrm{a}, \mathrm{H} 6}=14.9 \mathrm{~Hz}$ ), 165.2 (CO, $\left.{ }^{3} J_{\mathrm{CO}, \mathrm{H} 6}=2.3 \mathrm{~Hz}\right)$; MS m/z (\%): $227\left(\mathrm{M}^{+}, 33\right), 226\left(\mathrm{M}^{+}-1,29\right), 225\left(\mathrm{M}^{+}, 100\right), 224\left(\mathrm{M}^{+}-1,65\right)$.

5-Chloro-2-(methylthio)pyrimidine-4-carboxylic acid (4a). ${ }^{1} \mathrm{H}-\mathrm{NMR}$ (300 MHz, DMSO-d 6 ) $\delta: 2.51$ (3H, s, SMe), 7.40-10.20 (1H, br s, OH), 8.84 (1H, s, H-6); ${ }^{13} \mathrm{C}-\mathrm{NMR}$ (75 MHz, DMSO-d $\left.d_{6}\right): 14.0$ (SMe, $\left.{ }^{1} J=141.9 \mathrm{~Hz}\right), 121.5\left(\mathrm{C}-5,{ }^{2} J_{\mathrm{C} 5, \mathrm{H} 6}=3.8 \mathrm{~Hz}\right), 157.0$ (C-4), 158.2 (C-6, $\left.{ }^{1} J=191.7 \mathrm{~Hz}\right), 164.5$ (CO, $\left.{ }^{4} J_{\mathrm{CO}, \mathrm{H} 6}=1.6 \mathrm{~Hz}\right), 169.9\left(\mathrm{C}-2,{ }^{3} J_{\mathrm{C} 2, \mathrm{SMe}}=4.7 \mathrm{~Hz},{ }^{3} J_{\mathrm{C} 2, \mathrm{H} 6}=12.4 \mathrm{~Hz}\right) ;{ }^{15} \mathrm{~N}-\mathrm{NMR}\left(50 \mathrm{MHz}, \mathrm{DMSO}-d_{6}\right) \delta$ : $-102.5(\mathrm{~N}-3),-89.5(\mathrm{~N}-1)$. 
3-Chlorothieno[2,3-b]thiophene-2-carbonyl chloride (7b). ${ }^{1} \mathrm{H}-\mathrm{NMR}\left(300 \mathrm{MHz}, \mathrm{CDCl}_{3}\right) \delta$ : $7.31(1 \mathrm{H}$, d, $\left.{ }^{3} J_{\mathrm{H} 4, \mathrm{H} 5}=5.4 \mathrm{~Hz}, \mathrm{H}-4\right), 7.51\left(1 \mathrm{H}, \mathrm{d},{ }^{3} J_{\mathrm{H} 5, \mathrm{H} 4}=5.4 \mathrm{~Hz}, \mathrm{H}-5\right) ;{ }^{13} \mathrm{C}-\mathrm{NMR}\left(75 \mathrm{MHz}, \mathrm{CDCl}_{3}\right) \delta: 119.9(\mathrm{C}-4$, $\left.{ }^{1} J=175.5 \mathrm{~Hz},{ }^{2} J_{\mathrm{C} 4, \mathrm{H} 5}=4.1 \mathrm{~Hz}\right), 127.5\left(\mathrm{C}-3,{ }^{3} J_{\mathrm{C} 3, \mathrm{H} 4}=1.0 \mathrm{~Hz},{ }^{4} J_{\mathrm{C} 3, \mathrm{H} 5}=0.8 \mathrm{~Hz}\right), 131.0\left(\mathrm{C}-5,{ }^{1} \mathrm{~J}=189.0 \mathrm{~Hz}\right.$, $\left.{ }^{2} J_{\mathrm{C} 5, \mathrm{H} 4}=7.0 \mathrm{~Hz}\right), 131.5\left(\mathrm{C}-2,{ }^{5} J_{\mathrm{C} 2, \mathrm{H} 5}=1.0 \mathrm{~Hz}\right), 144.5$ (C-6a, $\left.{ }^{3} J_{\mathrm{C} 6 \mathrm{a}, \mathrm{H} 5}=7.8 \mathrm{~Hz},{ }^{3} J_{\mathrm{C} 6 \mathrm{a}, \mathrm{H} 4}=9.2 \mathrm{~Hz}\right), 146.3$ (C3a, $\left.{ }^{2} J_{\mathrm{C} 3 \mathrm{a}, \mathrm{H} 4}=4.9 \mathrm{~Hz},{ }^{3} J_{\mathrm{C} 3 \mathrm{a}, \mathrm{H} 5}=10.8 \mathrm{~Hz}\right), 156.8(\mathrm{CO})$; MS m/z (\%): $240\left(\mathrm{M}^{+}, 3\right), 238\left(\mathrm{M}^{+}, 14\right), 236\left(\mathrm{M}^{+}\right.$, 18), 203 (42), 201 (100), 173 (18), 93 (15), 69 (18).

7-Chlorothieno[2,3-f][1,3] benzodioxole-6-carbonyl chloride (8b). ${ }^{1} \mathrm{H}-\mathrm{NMR}\left(300 \mathrm{MHz}, \mathrm{CDCl}_{3}\right) \delta$ : $6.13(2 \mathrm{H}, \mathrm{s}, \mathrm{H}-2), 7.16\left(1 \mathrm{H}, \mathrm{d},{ }^{5} J_{\mathrm{H} 4, \mathrm{H} 8}=0.5 \mathrm{~Hz}, \mathrm{H}-4\right), 7.30\left(1 \mathrm{H}, \mathrm{d},{ }^{5} J_{\mathrm{H} 8, \mathrm{H} 4}=0.5 \mathrm{~Hz}, \mathrm{H}-8\right) ;{ }^{13} \mathrm{C}-\mathrm{NMR}(75$ $\left.\mathrm{MHz}, \mathrm{CDCl}_{3}\right) \delta$ : $101.2\left(\mathrm{C}-4,{ }^{1} \mathrm{~J}=169.7 \mathrm{~Hz}\right), 102.1$ (C-8, $\left.{ }^{1} \mathrm{~J}=169.7 \mathrm{~Hz}\right), 102.5$ (C-2, $\left.{ }^{1} \mathrm{~J}=175.0 \mathrm{~Hz}\right), 127.4$ (C-6), 130.0 (C-7, $\left.{ }^{3} J_{\mathrm{C} 7, \mathrm{H} 8}=3.8 \mathrm{~Hz}\right), 132.5$ (C-7a, $\left.{ }^{3} J_{\mathrm{C} 7 \mathrm{a}, \mathrm{H} 4}=6.3 \mathrm{~Hz}\right), 136.9$ (C-4a, $\left.{ }^{3} J_{\mathrm{C} 4 \mathrm{a}, \mathrm{H} 8}=7.4 \mathrm{~Hz}\right), 148.6$ $\left(\mathrm{C}-8 \mathrm{a},{ }^{2} J_{\mathrm{C} 8 \mathrm{a}, \mathrm{H} 8}=4.2 \mathrm{~Hz},{ }^{3} J_{\mathrm{C} 8 \mathrm{a}, \mathrm{H} 2}=1.8 \mathrm{~Hz},{ }^{3} J_{\mathrm{C} 8 \mathrm{a}, \mathrm{H} 4}=6.7 \mathrm{~Hz}\right), 151.5\left(\mathrm{C}-3 \mathrm{a},{ }^{2} J_{\mathrm{C} 3 \mathrm{a}, \mathrm{H} 4}=3.8 \mathrm{~Hz},{ }^{3} J_{\mathrm{C} 3 \mathrm{a}, \mathrm{H} 2}=1.7 \mathrm{~Hz}\right.$, $\left.{ }^{3} J_{\mathrm{C} 3 \mathrm{a}, \mathrm{H} 8}=7.2 \mathrm{~Hz}\right), 157.5(\mathrm{CO})$; MS m/z (\%): $278\left(\mathrm{M}^{+}, 3\right), 276\left(\mathrm{M}^{+}, 15\right), 274\left(\mathrm{M}^{+}, 17\right), 241$ (36), 239 (100), 211 (29), 201 (48), 69 (37).

Compounds 9, 10, 16-20: General Procedure.

Under anhydrous conditions, a solution/suspension of the corresponding $o$-haloarenecarbonyl chloride $\mathbf{2 b}-\mathbf{8 b}$ ( $3 \mathrm{mmol}$ ) in anhydrous 1,4-dioxane (3-5 $\mathrm{mL}$ ) was added to a suspension of pyrazolone 1 (523 mg, $3 \mathrm{mmol}$ ) and $\mathrm{Ca}(\mathrm{OH})_{2}$ (445 mg, $6 \mathrm{mmol}$ ) in anhydrous 1,4-dioxane (3-5 mL). The reaction mixture was heated at reflux under stirring for $3 \mathrm{~h}$. After cooling to room temperature, the mixture was acidified with $2 \mathrm{M} \mathrm{HCl}(5-7 \mathrm{ml})$, stirred for $15 \mathrm{~min}$, and poured into $\mathrm{H}_{2} \mathrm{O}$ (10-15 ml). After $30 \mathrm{~min}$, solid products were filtered off, washed with $\mathrm{H}_{2} \mathrm{O}$, and recrystallised. NMR data of compounds 9 and $\mathbf{1 0}$ are presented in Table 1.

6-Chloro-3-methyl-1-phenylpyrazolo[4',3':5,6]pyrano[2,3-c]pyridazin-4(1H)-one (9). Off-white needles (yield 66\%); Mp 209-212.5 ${ }^{\circ} \mathrm{C}$ (aqueous ethanol); IR (KBr) cm ${ }^{-1}: 1678$; MS m/z (\%): $315\left(\mathrm{M}^{+}\right.$ +1 , 6), $314\left(\mathrm{M}^{+}, 36\right), 313\left(\mathrm{M}^{+}-1,27\right), 312\left(\mathrm{M}^{+}, 100\right), 311\left(\mathrm{M}^{+}-1,45\right), 77$ (37); Anal. Calcd. for $\mathrm{C}_{15} \mathrm{H}_{9} \mathrm{ClN}_{4} \mathrm{O}_{2}$ (312.7106): C, 57.61; H, 2.90; N, 17.92. Found: C, 57.83; H, 3.12; N, 17.93.

1,3,7-Trimethyl-9-phenyl-3H-pyrazolo[3,4-b]pyrazolo[4',3':5,6] pyrano[2,3-d]pyridin-6(9H)-one (10). Colourless needles (yield 63\%); Mp 248.5-251 ${ }^{\circ} \mathrm{C}$ (aqueous ethanol); IR (KBr) $\mathrm{cm}^{-1}: 1667 ; \mathrm{MS} \mathrm{m} / \mathrm{z}$ (\%): $346\left(\mathrm{M}^{+}+1,19 \%\right), 345\left(\mathrm{M}^{+}, 100\right), 344\left(\mathrm{M}^{+}-1\right.$, 46); Anal. Calcd. for $\mathrm{C}_{19} \mathrm{H}_{15} \mathrm{~N}_{5} \mathrm{O}_{2}$ (345.3547): C, 66.08; H, 4.38; N, 20.28. Found: C, 65.83; H, 4.44; N, 20.35.

[5-Chloro-2-(methylthio)pyrimidin-4-yl](5-hydroxy-3-methyl-1-phenyl-1H-pyrazol-4-yl)methanone (16). Colourless needles (yield 59\%); Mp 212-217 ${ }^{\circ} \mathrm{C}$ (aqueous ethanol); ${ }^{1} \mathrm{H}-\mathrm{NMR}$ (500 MHz, DMSO- $d_{6}$ ) $\delta$ : 2.49 (3H, s, pyrazole 3-Me), 2.51 (3H, s, SMe), 7.00-10.00 (1H, br s, OH), 7.28 (1H, m, Ph H-4), 7.46 (2H, m, Ph H-3,5), 7.60 (2H, m, Ph H-2,6), 8.82 (1H, s, pyrimidine H-6); ${ }^{13} \mathrm{C}-\mathrm{NMR}$ (125 MHz, DMSO- $d_{6}$ ) $\delta$ : 13.7 (pyrazole 3-Me, ${ }^{1} J=130.3 \mathrm{~Hz}$ ), 14.0 (SMe, ${ }^{1} \mathrm{~J}=141.8 \mathrm{~Hz}$ ), 102.6 (pyrazole C4, ${ }^{3} J_{\mathrm{C} 4,3-\mathrm{Me}}=2.5 \mathrm{~Hz}$ ), 120.7 (Ph C-2,6), 121.6 (pyrimidine C-5, ${ }^{2} J_{\mathrm{C} 5, \mathrm{H} 6}=3.6 \mathrm{~Hz}$ ), 126.1 (Ph C-4), 129.0 
(Ph C-3,5), 135.8 (Ph C-1), 150.9 (pyrazole C-3, ${ }^{2} J_{\mathrm{C} 3,3-\mathrm{Me}}=6.7 \mathrm{~Hz}$ ), 156.8 (pyrimidine C-6, ${ }^{1} J=190.5$ $\mathrm{Hz}$ ), 160.0 (pyrazole C-5), 163.0 (pyrimidine C-4, ${ }^{3} J_{\mathrm{C} 4, \mathrm{H} 6}=3.7 \mathrm{~Hz}$ ), 169.5 (pyrimidine C-2, ${ }^{3} J_{\mathrm{C} 2, \mathrm{SMe}}=4.7$ $\mathrm{Hz},{ }^{3} J_{\mathrm{C} 2, \mathrm{H} 6}=12.1 \mathrm{~Hz}$ ), $182.8\left(\mathrm{CO},{ }^{4} J_{\mathrm{CO}, \mathrm{H} 6}=1.6 \mathrm{~Hz}\right.$ ); ${ }^{15} \mathrm{~N}-\mathrm{NMR}$ (50 MHz, DMSO- $d_{6}$ ) $\delta:-201.9$ (pyrazole $\mathrm{N}-1$ ), -102.0 (pyrimidine $\mathrm{N}-3$ ), -93.2 (pyrimidine $\mathrm{N}-1$ ); the pyrazole $\mathrm{N}-2$ was not found; IR (KBr) $\mathrm{cm}^{-1}$ : 1630; MS m/z (\%): 362 (M+15), 360 (M+1 39), 326 (20), 325 (100), 201 (32), 159 (41), 91 (22), 77 (45); Anal. Calcd. for $\mathrm{C}_{16} \mathrm{H}_{13} \mathrm{ClN}_{4} \mathrm{O}_{2} \mathrm{~S}$ (360.8180): C, 53.26; H, 3.63; N, 15.53. Found: C, 53.38; H, 3.78; N, 15.40 .

(2-Bromo-3-thienyl)(5-hydroxy-3-methyl-1-phenyl-1H-pyrazol-4-yl)methanone (17). Almost colourless crystals (yield 73\%); Mp 131-133 ${ }^{\circ} \mathrm{C}$ (aqueous ethanol); ${ }^{1} \mathrm{H}-\mathrm{NMR}\left(300 \mathrm{MHz}, \mathrm{CDCl}_{3}\right) \delta$ : 2.05 (3H, s, Me), $7.04\left(1 \mathrm{H}, \mathrm{d},{ }^{3} J_{\mathrm{H} 4, \mathrm{H} 5}=5.7 \mathrm{~Hz}\right.$, thiophene $\left.\mathrm{H}-4\right), 7.31(1 \mathrm{H}, \mathrm{m}, \mathrm{Ph} \mathrm{H}-4), 7.38\left(1 \mathrm{H}, \mathrm{d},{ }^{3} J_{\mathrm{H} 5, \mathrm{H} 4}=5.7 \mathrm{~Hz}\right.$, thiophene H-5), 7.47 (2H, m, Ph H-3,5), 7.86 (2H, m, Ph H-2,6), 8.81 (1H, s, OH); ${ }^{13} \mathrm{C}-\mathrm{NMR}(75$ $\mathrm{MHz}, \mathrm{CDCl}_{3}$ ) $\delta: 14.2\left(\mathrm{Me},{ }^{1} \mathrm{~J}=128.9 \mathrm{~Hz}\right.$ ), 104.7 (pyrazole C-4, ${ }^{3} \mathrm{~J}_{\mathrm{C} 4, \mathrm{Me}}=2.6 \mathrm{~Hz}$ ), 113.0 (thiophene C-2, ${ }^{3} J_{\mathrm{C} 2, \mathrm{H} 5}=7.6 \mathrm{~Hz},{ }^{3} J_{\mathrm{C} 2, \mathrm{H} 4}=12.4 \mathrm{~Hz}$ ), 120.9 (Ph C-2,6), 126.9 (Ph C-4), 127.1 (thiophene C-4, ${ }^{1} \mathrm{~J}=172.2 \mathrm{~Hz}$, ${ }^{2} J_{\mathrm{C} 4, \mathrm{H} 5}=4.2 \mathrm{~Hz}$ ), 127.5 (thiophene C-5, $\left.{ }^{1} J=189.0 \mathrm{~Hz},{ }^{2} J_{\mathrm{C} 5, \mathrm{H} 4}=6.8 \mathrm{~Hz}\right), 129.1(\mathrm{Ph} \mathrm{C}-3,5), 137.1$ (Ph C-1), 139.2 (thiophene C-3, ${ }^{2} J_{\mathrm{C} 3, \mathrm{H} 4}=5.4 \mathrm{~Hz},{ }^{3} J_{\mathrm{C} 3, \mathrm{H} 5}=8.7 \mathrm{~Hz}$ ), 148.3 (pyrazole C-3, ${ }^{2} J_{\mathrm{C} 3, \mathrm{Me}}=7.0 \mathrm{~Hz}$ ), 160.3 (pyrazole C-5), 186.8 (CO); ${ }^{15} \mathrm{~N}-\mathrm{NMR}\left(50 \mathrm{MHz}, \mathrm{CDCl}_{3}\right) \delta:-190.3(\mathrm{~N}-1),-100.6$ (N-2); IR (KBr)

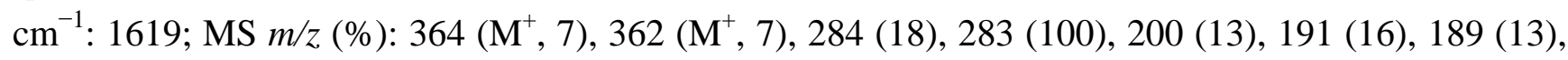
91 (17), 77 (26), 67 (24); Anal. Calcd. for $\mathrm{C}_{15} \mathrm{H}_{11} \mathrm{BrN}_{2} \mathrm{O}_{2} \mathrm{~S}$ (363.2290): C, 49.60; H, 3.05; N, 7.71. Found: C, 49.63; H, 3.20; N, 7.69.

(4-Bromo-3-thienyl)(5-hydroxy-3-methyl-1-phenyl-1H-pyrazol-4-yl)methanone (18). Almost colourless crystals (yield 65\%); Mp 120-121 ${ }^{\circ} \mathrm{C}$ (aqueous ethanol); ${ }^{1} \mathrm{H}-\mathrm{NMR}\left(300 \mathrm{MHz}, \mathrm{CDCl}_{3}\right) \delta$ : 2.03 (3H, s, Me), 7.32 (1H, m, Ph H-4), $7.40\left(1 \mathrm{H}, \mathrm{d},{ }^{3} J_{\mathrm{H} 5, \mathrm{H} 2}=3.3 \mathrm{~Hz}\right.$, thiophene H-5), 7.47 (2H, m, Ph H-3,5), 7.56 $\left(1 \mathrm{H}, \mathrm{d},{ }^{3} J_{\mathrm{H} 2 \mathrm{H} 5}=3.3 \mathrm{~Hz}\right.$, thiophene H-2), $7.86(2 \mathrm{H}, \mathrm{m}, \mathrm{Ph} \mathrm{H}-2,6), 9.25$ (1H, s, OH); ${ }^{13} \mathrm{C}-\mathrm{NMR}(75 \mathrm{MHz}$, $\mathrm{CDCl}_{3}$ ) $\delta$ : $14.5\left(\mathrm{Me},{ }^{1} \mathrm{~J}=128.9 \mathrm{~Hz}\right.$ ), 104.8 (pyrazole C-4, ${ }^{3} J_{\mathrm{C} 4, \mathrm{Me}}=2.6 \mathrm{~Hz}$ ), 108.7 (thiophene C-4, ${ }^{2} J_{\mathrm{C} 4, \mathrm{H} 5}=1.0 \mathrm{~Hz},{ }^{3} J_{\mathrm{C} 4, \mathrm{H} 2}=11.9 \mathrm{~Hz}$ ), 120.9 (Ph C-2,6), 125.0 (thiophene C-5, ${ }^{1} \mathrm{~J}=192.8 \mathrm{~Hz},{ }^{3} J_{\mathrm{C} 5, \mathrm{H} 2}=4.3$ $\mathrm{Hz}$ ), 126.9 (Ph C-4), 127.7 (thiophene C-2, $\left.{ }^{1} \mathrm{~J}=189.3 \mathrm{~Hz},{ }^{3} J_{\mathrm{C} 2, \mathrm{H} 5}=5.7 \mathrm{~Hz}\right), 129.1$ (Ph C-3,5), 137.0 (Ph C-1), 139.3 (thiophene C-3, ${ }^{2} J_{\mathrm{C} 3, \mathrm{H} 2}=4.2 \mathrm{~Hz},{ }^{3} J_{\mathrm{C} 3, \mathrm{H} 5}=8.0 \mathrm{~Hz}$ ), 148.3 (pyrazole C-3, ${ }^{2} J_{\mathrm{C} 3, \mathrm{Me}}=6.9 \mathrm{~Hz}$ ), 160.2 (pyrazole C-5), $186.6\left(\mathrm{CO},{ }^{3} J_{\mathrm{CO}, \mathrm{H} 2}=2.6 \mathrm{~Hz}\right) ;{ }^{15} \mathrm{~N}-\mathrm{NMR}\left(50 \mathrm{MHz}, \mathrm{CDCl}_{3}\right) \delta:-190.2(\mathrm{~N}-1)$, -100.7 (N-2); IR (KBr) cm ${ }^{-1}$ : 1602; MS m/z (\%): 364 (M+4, 42), 362 (M+4 41), 283 (100), 200 (74), 191 (39), 189 (33), 91 (40), 77 (36), 69 (36), 67 (35); Anal. Calcd. for $\mathrm{C}_{15} \mathrm{H}_{11} \mathrm{BrN}_{2} \mathrm{O}_{2} \mathrm{~S}$ (363.2290): C, 49.60; H, 3.05; N, 7.71; S, 8.83. Found: C, 49.79; H, 2.88; N, 7.51; S, 8.66.

(3-Chlorothieno[2,3-b]thien-2-yl)(5-hydroxy-3-methyl-1-phenyl-1H-pyrazol-4-yl)methanone (19).

Yellowish crystals (yield 54\%); Mp 193-197 ${ }^{\circ} \mathrm{C}$ (aqueous ethanol); ${ }^{1} \mathrm{H}-\mathrm{NMR}$ (300 MHz, DMSO- $d_{6}$ ) $\delta$ : 2.29 (3H, s, Me), $4.32(1 \mathrm{H}, \mathrm{s}, \mathrm{OH}), 7.30(1 \mathrm{H}, \mathrm{m}, \mathrm{Ph} \mathrm{H}-4), 7.32\left(1 \mathrm{H}, \mathrm{d},{ }^{3} J_{\mathrm{H} 4, \mathrm{H} 5}=5.4 \mathrm{~Hz}\right.$, thienoth. H-4), 7.47 (2H, m, Ph H-3,5), 7.68 (2H, m, Ph H-2,6), 7.78 (1H, d, ${ }^{3} \mathrm{~J}_{\mathrm{H} 5, \mathrm{H} 4}=5.4 \mathrm{~Hz}$, thienoth. $\left.\mathrm{H}-5\right)$; ${ }^{13} \mathrm{C}-\mathrm{NMR}$ (75 MHz, DMSO- $d_{6}$ ) $\delta: 13.4$ (Me, ${ }^{1} J=129.2$ ), 105.2 (pyrazole C-4, ${ }^{3} J_{\mathrm{C} 4, \mathrm{Me}}=2.7 \mathrm{~Hz}$ ), 118.0 (thienoth. C$\left.3,{ }^{3} J_{\mathrm{C} 3, \mathrm{H} 4}=1.0 \mathrm{~Hz},{ }^{4} J_{\mathrm{C} 3, \mathrm{H} 5}=0.9 \mathrm{~Hz}\right), 118.9$ (thienoth. C-4, $\left.{ }^{1} \mathrm{~J}=173.3 \mathrm{~Hz},{ }^{2} J_{\mathrm{C} 4, \mathrm{H} 5}=4.8 \mathrm{~Hz}\right), 121.2(\mathrm{Ph} \mathrm{C}-$ 2,6), 126.1 (Ph C-4), 129.0 (Ph C-3,5), 131.9 (thienoth. C-5, ${ }^{1} J=189.3 \mathrm{~Hz},{ }^{2} J_{\mathrm{C} 5, \mathrm{H} 4}=7.4 \mathrm{~Hz}$ ), 136.9 (Ph C-1), 138.9 (thienoth. C-6a, ${ }^{3} J_{\mathrm{C} 6 \mathrm{a}, \mathrm{H} 5}=8.0 \mathrm{~Hz},{ }^{3} J_{\mathrm{C} 6 \mathrm{a}, \mathrm{H} 4}=9.1 \mathrm{~Hz}$ ), 139.2 (thienoth. C-2, ${ }^{5} J_{\mathrm{C} 2, \mathrm{H} 5}=0.8 \mathrm{~Hz}$ ), 
144.3 (thienoth. C-3a, ${ }^{2} J_{\mathrm{C} 3 \mathrm{a}, \mathrm{H} 4}=5.1 \mathrm{~Hz},{ }^{3} J_{\mathrm{C} 3 \mathrm{a}, \mathrm{H} 5}=10.6 \mathrm{~Hz}$ ), 149.6* (pyrazole C-3), 156.4* (pyrazole C5), 179.7 (CO). * broad signal; MS m/z (\%): $376\left(\mathrm{M}^{+}, 2\right), 374\left(\mathrm{M}^{+}, 7\right), 339$ (38), 218 (21), 201 (36), 200 (100), 91 (33); Anal. Calcd. for $\mathrm{C}_{17} \mathrm{H}_{11} \mathrm{ClN}_{2} \mathrm{O}_{2} \mathrm{~S}_{2}$ (374.8644): C, 54.47; H, 2.96; N, 7.47. Found: C, 54.24; H, 3.04; N, 7.45.

(7-Chlorothieno[2,3-f][1,3] benzodioxol-6-yl)(5-hydroxy-3-methyl-1-phenyl-1H-pyrazol-4-yl)methanone (20). Dark red powder (yield 93\%); Mp 143-147.5 ${ }^{\circ} \mathrm{C} ;{ }^{1} \mathrm{H}-\mathrm{NMR}$ (300 MHz, DMSO- $d_{6}$ ) $\delta$ : $2.28(3 \mathrm{H}, \mathrm{s}, \mathrm{Me}), 5.32(1 \mathrm{H}, \mathrm{s}, \mathrm{OH}), 6.17(2 \mathrm{H}, \mathrm{s}$, tricycle $\mathrm{H}-2), 7.29(1 \mathrm{H}, \mathrm{m}, \mathrm{Ph} \mathrm{H}-4), 7.29(1 \mathrm{H}, \mathrm{s}$, tricycle H-8), 7.47 (2H, m, Ph H-3,5), 7.62 (1H, s, tricycle H-4), 7.67 (2H, m, Ph H-2,6); ${ }^{13} \mathrm{C}-\mathrm{NMR}(75$ MHz, DMSO- $d_{6}$ ) $\delta$ : 13.5 (Me, ${ }^{1} J=129.3 \mathrm{~Hz}$ ), 100.7 (tricycle C-8, ${ }^{1} \mathrm{~J}=168.1 \mathrm{~Hz}$ ), 102.1 (tricycle C-4, ${ }^{1} J=170.6$ ), 102.2 (tricycle C-2, ${ }^{1} J=176.3$ ), 105.4 (pyrazole C-4, ${ }^{3} J_{\mathrm{C} 4, \mathrm{Me}}=2.5 \mathrm{~Hz}$ ), 120.1 (tricycle C-7, ${ }^{3} J_{\mathrm{C} 7 \mathrm{H} 8}=3.7 \mathrm{~Hz}$ ), 121.2 (Ph C-2,6), 126.2 (Ph C-4), 129.0 (Ph C-3,5), 130.9 (tricycle C-7a, ${ }^{3} J_{\mathrm{C} 7 \mathrm{a}, \mathrm{H} 4}=6.3$ ), 132.3 (tricycle C-4a, ${ }^{2} J_{\mathrm{C} 4 \mathrm{a}, \mathrm{H} 4}=1.6 \mathrm{~Hz},{ }^{3} \mathrm{~J}_{\mathrm{C} 4 \mathrm{a}, \mathrm{H} 8}=7.7 \mathrm{~Hz}$ ), 134.7 (tricycle C-6), 136.7 (Ph C-1), 147.8 (tricycle C-8a, ${ }^{2} J_{\mathrm{C} 8 \mathrm{a}, \mathrm{H} 8}=3.8 \mathrm{~Hz},{ }^{3} J_{\mathrm{C} 8 \mathrm{a}, \mathrm{H} 4}=6.9 \mathrm{~Hz}$ ), 148.9 (tricycle C-3a, ${ }^{2} J_{\mathrm{C} 3 \mathrm{a}, \mathrm{H} 4}=3.5 \mathrm{~Hz},{ }^{3} J_{\mathrm{C} 3 \mathrm{a}, \mathrm{H} 8}=7.0 \mathrm{~Hz}$ ), 149.7 (pyrazole C-3, ${ }^{2} J_{\mathrm{C} 3, \mathrm{Me}}=7.1 \mathrm{~Hz}$ ), 156.7 (pyrazole C-5), 180.1 (CO); MS m/z (\%): 414 (M+1 3), 412 (M+, 7), 378 (22), 377 (100), 256 (28), 212 (53), 211 (26), 200 (67), 91 (31); Anal. Calcd. for $\mathrm{C}_{20} \mathrm{H}_{13} \mathrm{ClN}_{2} \mathrm{O}_{4} \mathrm{~S}(412.8462) \cdot 0.1 \mathrm{H}_{2} \mathrm{O}$ : C, 57.68; H, 3.24; N, 6.73. Found: C, 57.61; H, 3.38; N, 6.53.

Cyclisation of 4-Aroylpyrazol-5-ols 16-20 into Compounds 11-15; General Procedure.

Under anhydrous conditions, the corresponding 4-aroylpyrazol-5-ol 16-20 (1 mmol) was dissolved/suspended in anhydrous DMF ( $5 \mathrm{~mL}$ ) and added to a suspension of $\mathrm{NaH}(60 \%$ in mineral oil; $1 \mathrm{mmol}$ ) in anhydrous DMF (5 mL). The reaction mixture was heated at reflux overnight and the solvent was then removed under reduced pressure. $\mathrm{H}_{2} \mathrm{O}(5 \mathrm{~mL})$ was added to the residue and stirring was continued for $1 \mathrm{~h}$ further. The precipitate formed was filtered off, washed with $\mathrm{H}_{2} \mathrm{O}$ and petroleum ether and recrystallised. NMR data of compounds 11-15 are presented in Table 1.

3-Methyl-6-(methylthio)-1-phenylpyrazolo[4',3':5,6]pyrano[3,2-d]pyrimidin-4(1H)-one (11). Colourless needles (yield 67\%); Mp 242.5-244.5 ${ }^{\circ} \mathrm{C}$ (aqueous ethanol); IR (KBr) $\mathrm{cm}^{-1}$ : 1672; MS m/z (\%): $325\left(\mathrm{M}^{+}+1\right.$, 18), $324\left(\mathrm{M}^{+}, 100\right), 77$ (22); Anal. Calcd. for $\mathrm{C}_{16} \mathrm{H}_{12} \mathrm{~N}_{4} \mathrm{O}_{2} \mathrm{~S}$ (324.3571): C, 59.25; H, 3.73; N, 17.27. Found: C, 59.29; H, 3.86; N, 17.06.

3-Methyl-1-phenylthieno[3',2':5,6]pyrano[2,3-c]pyrazol-4(1H)-one (12). Colourless needles (yield 92\%); Mp 176-179 ${ }^{\circ} \mathrm{C}$ (aqueous ethanol); IR (KBr) $\mathrm{cm}^{-1}$ : 1642; MS m/z (\%): $283\left(\mathrm{M}^{+}+1,22\right), 282$ $\left(\mathrm{M}^{+}, 100\right), 281\left(\mathrm{M}^{+}-1,58\right), 77$ (28); Anal. Calcd. for $\mathrm{C}_{15} \mathrm{H}_{10} \mathrm{~N}_{2} \mathrm{O}_{2} \mathrm{~S}$ (282.3171): C, 63.81; H, 3.57; N, 9.92. Found: C, 63.65; H, 3.79; N, 9.74.

3-Methyl-1-phenylthieno[3',4':5,6]pyrano[2,3-c]pyrazol-4(1H)-one (13). Beige powder (yield $\leq 5 \%$ ); Mp 138-145 ${ }^{\circ} \mathrm{C}$; MS m/z (\%): $283\left(\mathrm{M}^{+}+1,24\right), 282\left(\mathrm{M}^{+}, 100\right), 281\left(\mathrm{M}^{+}-1,79\right), 77$ (27); HRMS m/z: 282.0456 (Calcd for $\mathrm{C}_{15} \mathrm{H}_{10} \mathrm{~N}_{2} \mathrm{O}_{2} \mathrm{~S}:$ 282.0463). 
3-Methyl-1-phenylthieno[3",2":4',5'] thieno[2',3':5,6]pyrano[2,3-c]pyrazol-4(1H)-one (14). Slightly yellowish needles (yield 90\%); Mp 239-243 ${ }^{\circ} \mathrm{C}$; IR (KBr) $\mathrm{cm}^{-1}: 1646$; MS m/z (\%): $339\left(\mathrm{M}^{+}+1,21\right)$, $338\left(\mathrm{M}^{+}, 100\right), 337\left(\mathrm{M}^{+}-1\right.$, 45), 77 (18); Anal. Calcd. for $\mathrm{C}_{17} \mathrm{H}_{10} \mathrm{~N}_{2} \mathrm{O}_{2} \mathrm{~S}_{2}$ (338.4035): C, 60.34; $\mathrm{H}$, 2.98; N, 8.28. Found: C, 60.15; H, 3.27; N, 8.28.

3-Methyl-1-phenyl[1,3]dioxolo[5',6'][1] benzothieno[2',3':5,6]pyrano[2,3-c]pyrazol-4(1H)-one (15). Almost colourless needles (yield 80\%); Mp 324-327 ${ }^{\circ} \mathrm{C}$ (toluene); IR (KBr) cm ${ }^{-1}: 1653 ; \mathrm{MS} \mathrm{m} / \mathrm{z}(\%)$ : $377\left(\mathrm{M}^{+}+1\right.$, 35), $376\left(\mathrm{M}^{+}, 100\right), 375\left(\mathrm{M}^{+}-1\right.$, 38), 91 (22), 77 (27); Anal. Calcd. for $\mathrm{C}_{20} \mathrm{H}_{12} \mathrm{~N}_{2} \mathrm{O}_{4} \mathrm{~S}$ (376.3853): C, 63.82; H, 3.21; N, 7.44. Found: C, 63.59; H, 3.42; N, 7.33.

\section{Acknowledgements}

We are grateful to Dr. Csaba Szántay Jr. for providing a pulse sequence for the ${ }^{13} \mathrm{C}\left\{{ }^{1} \mathrm{H}\right\}$ NOEdifference experiments and to Dr. L. Jirovetz for recording the mass spectra.

\section{References}

1. Carey, J. S.; Laffan, D.; Thomson, C.; Williams, M. T. Analysis of the reactions used for the preparation of drug candidate molecules. Org. Biomol. Chem. 2006, 4, 2337-2347.

2. Abraham, D. J., ed.; Burger's Medicinal Chemistry and Drug Discovery, $6^{\text {th }}$ ed.; WileyInterscience: New York, 2003; [Chem. Abstr. 2005, 143, 109740].

3. Makriyannis, A.; Biegel, D. Drug Discovery Strategies and Methods; Marcel Dekker, Inc.: New York, 2004; [Chem. Abstr. 2004, 140, 229394].

4. Eller, G. A.; Wimmer, V.; Haring, A. W.; Holzer, W. An Efficient Approach to Heterocyclic Analogues of Xanthone: A Short Synthesis of all possible Pyrido[5,6]pyrano[2,3-c]pyrazol4(1H)-ones. Synthesis 2006, 4219-4229.

5. Eller, G. A.; Haring, A. W.; Datterl, B.; Zwettler, M.; Holzer, W. Tri- and Tetracyclic Heteroaromatic Systems: Synthesis of Novel Benzo-, Benzothieno- and Thieno-Fused Pyrano[2,3-c]pyrazol-4(1H)-ones. Heterocycles 2007, 71, 87-104.

6. Eller, G. A.; Datterl, B.; Holzer, W.; manuscript submitted.

7. Kleemann, A.; Engel, J.; Kutscher, B.; Reichert, D. Pharmaceutical Substances: Syntheses, Patents, Applications, $4^{\text {th }}$ ed.; Thieme: Stuttgart, 2001; p. 99.

8. Bell, J. Amlexanox for the Treatment of Recurrent Aphthous Ulcers. Clin. Drug Invest. 2005, 25, 555-566.

9. Colotta, V.; Cecchi, L.; Catarzi, D.; Melani, F.; Filacchioni, G.; Martini, C.; Tacchi, P.; Lucacchini, A. 1-(3-Aminophenyl)-3-methyl[1]benzopyrano[2,3-c]pyrazol-4-one: a new selective A2 adenosine receptor antagonist. Pharm. Pharmacol. Lett. 1992, 2, 74-76; [Chem. Abstr. 1993, 118, 32807].

10. Eller, G. A.; Holzer, W. The 4-Methoxybenzyl (PMB) Function as a Versatile Protecting Group in the Synthesis of $N$-Unsubstituted Pyrazolones. Heterocycles 2004, 63, 2537-2555.

11. Holzer, W.; Eller, G. A.; Wimmer, V.; Haring, A.; manuscript in preparation. 
12. Minkin, V. I.; Garnovskii, A. D.; Elguero, J.; Katritzky, A. R.; Denisko, O. V. The tautomerism of heterocycles: Five-membered rings with two or more heteroatoms. Adv. Heterocycl. Chem. 2000, 76, 157-323; [Chem. Abstr. 2000, 133, 309576].

13. Jensen, B. S. Synthesis of 1-phenyl-3-methyl-4-acyl-5-pyrazolones. Acta Chem. Scand. 1959, 13, 1668-1670; [Chem. Abstr. 1962, 56, 66890].

14. Williams, A. Recent advances in NMR prediction and automated structure elucidation software. Curr. Opin. Drug Discovery Dev. 2000, 3, 298-305; [Chem. Abstr. 2000, 133, 222096].

15. NMRPredict, version 3.2.8; Modgraph Consultants, Ltd.: Herts, United Kingdom, 2004; www.modgraph.co.uk.

16. ACD/C+H NMR Predictors and DB, version 10.04; Advanced Chemistry Development, Inc.: Toronto, ON, Canada, 2006; www.acdlabs.com.

17. Braun, S.; Kalinowski, H.-O.; Berger, S. 150 and More Basic NMR Experiments: A Practical Course, $2^{\text {nd }}$ ed.; Wiley-VCH: Weinheim, 1998; [Chem. Abstr. 1999, 131, 184497].

18. Neuhaus, D.; Williamson, M. P. The Nuclear Overhauser Effect in Structural and Conformational Analysis, $2^{\text {nd }}$ ed.; Wiley-VCH, Inc.: New York, 2000; [Chem. Abstr. 2004, 142, 392075].

19. Kover, K. E.; Batta, G. Theoretical and practical aspects of one- and two-dimensional heteronuclear overhauser experiments and selective ${ }^{13} \mathrm{C} \quad T_{1}$-determinations of heteronuclear distances., Prog. Nucl. Magn. Reson. Spectrosc. 1987, 19, 223-266; [Chem. Abstr. 1987, 107, 145869].

20. Szántay, C, Jr.; Csepregi, Z.; Aranyosi, P.; Ruznák, I.; Töke, L.; Víg, A. Nuclear Magnetic Resonance Investigations of the Azo-Hydrazone Tautomerism of Azoreactive Dye Chromophores, Magn. Reson. Chem. 1997, 35, 306-310; [Chem. Abstr. 1997, 126, 294555].

21. Davis, D. G.; Bax, A. Simplification of proton NMR spectra by selective excitation of experimental subspectra. J. Am. Chem. Soc. 1985, 107, 7197-7198.

22. Sarkar, S. K.; Bax, A. A simple and sensitive one-dimensional NMR technique for correlation of proton and carbon chemical shifts. J. Magn. Reson. 1985, 62, 109-112.

23. Bax, A. Structure determination and spectral assignment by pulsed polarization transfer via longrange proton-carbon-13 couplings. J. Magn. Reson. 1984, 57, 314-318.

24. Jippo, T.; Kamo, O.; Nagayama, N. Determination of long-range proton-carbon 13 coupling constants with selective two-dimensional INEPT. J. Magn. Reson. 1986, 66, 344-348.

25. Willker, W.; Leibfritz, D.; Kerssebaum, R.; Bermel, W. Gradient selection in inverse heteronuclear correlation spectroscopy. Magn. Reson. Chem. 1993, 31, 287-292.

26. ACD/Name, version 10.01; Advanced Chemistry Development, Inc.: Toronto, ON, Canada, 2006; www.acdlabs.com.

27. Eller, G. A. Improving the Quality of Published Chemical Names with Nomenclature Software. Molecules 2006, 11, 915-928.

Sample Availability: Contact the authors.

(C) 2007 by MDPI (http://www.mdpi.org). Reproduction is permitted for non-commercial purposes. 